\title{
Écologie de deux Aedes halophiles, Aedes (O.) caspius (Pallas, 1771) et Aedes (O.) detritus (Haliday, 1833) dans le midi de la France
}

\section{I. - Structure chorionique et critères d'identification des deux espèces au stade œuf}

\author{
par A. GABINAUD, H. CRoseT, J. COUSSERANS et J.-A. RIOUX \\ Entente interdépartementale pour la Démoustication du Littoral méditerranéen, \\ rue P.-Rimbaud, F 34000 Montpellier, \\ Laboratoire de Zoologie et Biologie animale, Faculté des Sciences, \\ avenue de Villeneuve, $F 66000$ Perpignan, \\ Laboratoire d'Ecologie médicale et de Pathologie parasitaire, \\ et Institut de Botanique, rue A.-Broussonnet, F 34000 Montpellier.
}

\section{Résumé.}

Les auteurs décrivent la structure chorionique de l'œuf chez Aedes $(O$.$) caspius (Pallas,$ 1771) et Aedes (O.) detritus (Haliday, 1833). En corollaire, ils proposent une technique d'identification rapide basée sur l'emploi du microscope à contraste interférentiel en lumière réfléchie. La simplicité de la méthode rend possible l'inventaire systématique des gîtes de ponte et, partant, la cartographie écologique de ces gîtes.

\section{Summary.}

Ecology of two salt marshes mosquitoes in the South of France : Aedes (O.) caspius (Pallas, 1771) and Aedes (O.) detritus (Haliday, 1883). 1. - Chorionic pattern of eggs and quick technique for eggs identification.

The authors describe the chorionic patterns of eggs of Aedes (O.) caspius (Pallas, 1771) and Aedes $(O$.$) detritus (Haliday, 1833). They present a quick technique for their$ identification by the use of the interference contrast microscope with reflected light. This quite simple method make possible the inventory of the breeding places and, therefore, their ecological cartography. 
Dès 1926, l'étude de la structure et de l'ornementation chorionique des œufs d'Anophèles amenait P. Falleroni à faire éclater le «complexe maculipennis», amorçant les importants travaux des malariologistes italiens. Trente ans plus tard, W. R. Horsfall (1956) appliquait la technique aux œufs d'Aèdes, matériel jusqu'alors inaccessible à l'observation courante en raison de l'opacité du chorion.

Au demeurant, si de telles recherches visaient à rendre plus efficaces les méthodes de lutte, par la suite, d'autres motivations, de caractère fondamental, devaient s'y ajouter (dynamique des populations, déterminisme écologique de la ponte, etc...). C'est dans cette double perspective que nous avons entrepris l'identification des œufs de deux espèces d'Aèdes abondants dans le «midi » méditerranéen : Aedes $(O$. caspius (Pallas, 1771) et Aedes (O.) detritus (Haliday, 1833).

Chez ces espèces, le comportement de ponte est réglé par une série de tactismes qui lient l'espèce à certaines phytocénoses (J. A. Rioux, 1958). Lors du retrait des eaux, les œufs sont déposés sur le substrat de groupements végétaux caractéristiques (« enganes», jonchaies, roselières) qui peuvent ainsi servir « d'indicateurs » des gîtes de ponte et partant, à l'image des biotopes larvaires, permettre la levée de cartes écologiques (J. A. Rioux et coll., 1967 et 1968 ; J. Cousserans et coll., 1969). Pour ce faire, il est donc nécessaire de préciser au préalable, les liaisons statistiques existant entre les phytocénoses ainsi définies et les pontes correspondantes: autrement dit, de s'appuyer sur un nombre important d'échantillons convenablement choisis. Par ailleurs, étant donnée la masse considérable d'œufs présents dans chaque prélèvement, il est indispensable d'utiliser une technique rapide d'identification et de comptage.

La présente note comporte ainsi deux parties. Le premier chapitre traite de la structure chorionique; l'énoncé d'une technique rapide d'échantillonnage, basée sur les résultats précédents, fait l'objet du deuxième chapitre.

\section{Description}

Les œufs de référence proviennent d'imagos $q$ capturés en «Petite Camargue 》 au lieu-dit « Brasinvers» près d'Aigues-Mortes (Gard, France). Pondus sur substrat humide, ils sont maintenus pendant trois semaines à $20^{\circ} \mathrm{C}$, puis stockés à $15^{\circ} \mathrm{C}$ et $100 \%$ d'humidité.

Deux modes d'observation sont utilisés conjointement: le contraste de phase en lumière transmise, le contraste interférentiel en lumière réfléchie.

a) Examen en lumière transmise: les œufs sont préparés suivant la technique de G. B. Craig (1955): préalablement débarrassés de l'exochorion par frottement sur papier filtre humide, ils sont disséqués à l'aide de fines aiguilles. Après ablation de la calotte antérieure, la larve et la membrane vitelline en sont extraites. La coquille, placée dans une lame creuse, est alors décolorée par le chlore naissant: deux à trois gouttes d'acide chlorhydrique concentré projetées sur des cristaux de 
chlorate de potassium libèrent le chlore qui éclaircit et assouplit l'œuf en quelques minutes.

Après lavage et déshydratation, les fragments peuvent être montés directement entre lame et lamelle dans une goutte de baume du Canada. Il est possible également, d'étaler la coquille après avoir découpé l'extrémité postérieure et incisé le cylindre obtenu, selon une génératrice: pour réaliser cette opération, on utilise des ciseaux à cataracte dont l'une des lames a été aiguisée sous la loupe ; le cylindre est catétérisé sur la lame, puis sectionné. Transféré dans une goutte de baume, il est ensuite étalé et recouvert d'une lamelle. Cette technique est préférable à la précédente car elle permet la comparaison des diverses faces de l'œuf.
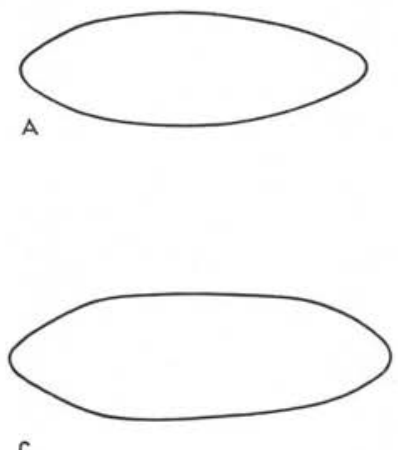

C
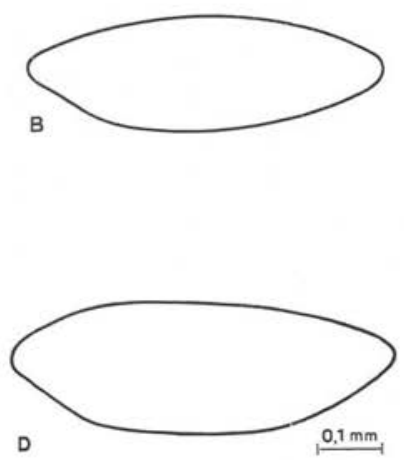

FIG. 1. - Silhouette de l'œuf chez Aedes (O.) caspius (Pallas, 1771) (A, B) et Aedes (O.) detritus (Haliday, 1883) (C, D). A et C, vue dorso-ventrale; B et $\mathrm{D}$, vue latérale.

L'examen est réalisé à l'aide du microscope à contraste de phase (Leitz Ortholux). Les clichés photographiques sont obtenus sur film Kodak microfile (objectif à immersion 70 ; temps de pose : 10 à 15 secondes).

b) Examen en lumière réfléchie: les œufs, préalablement débarrassés de l'exochorion, sont collés par une extrémité à la pointe de l'aiguille d'un insectoscope. Ce montage rend aisée l'observation de l'œuf sur chacune de ses faces.

L'examen est réalisé à l'aide du microscope à contraste interférentiel de Nomarski (Nachet 300) équipé de l'illuminateur épiscopique. L'objet peut être examiné sans autre préparation, à 5 grossissements différents $(130 \times$ à $700 \times)$. Malgré le fort pouvoir réflézhissant de l'œuf, l'image est bien contrastée de sorte que l'ornementation chorionique apparaît toujours nettement. Les clichés photographiques sont obtenus sur film Ilford FP4 (objectif à sec 50 ; temps de pose : 1 seconde).

Aedes (Ochlerotatus) caspius (Pallas, 1771).

Forme: Euf allongé, fusiforme dans sa partie postérieure. Asymétrie dorsoventrale discrète (fig. $1 \mathrm{~A}$ et $1 \mathrm{~B}$ ). 
TAllle: Longueur: $0,591 \pm 0,008 \mathrm{~mm}$ (nombre de mesures 100 ; variance 0,00141). Largeur : 0,189 $\pm 0,003 \mathrm{~mm}$ (nombre de mesures 100 ; variance 0,00016).

Couleur: Marron foncé à noir dense.

EXOcHORION : Epais, irrégulièrement adhérent.
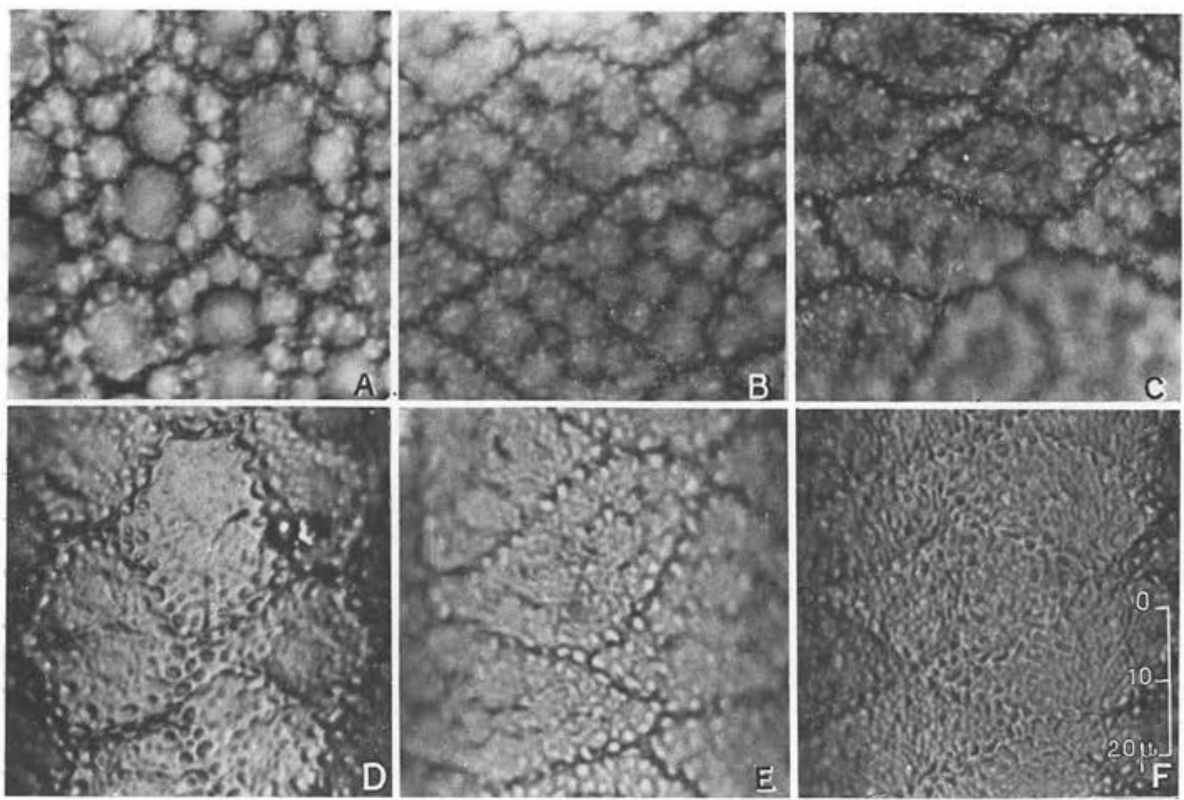

FIG. 2. - Ornementation chorionique de l'œuf chez Aedes (O.) caspius (Pallas, 1771). Vue en lumière transmise (contraste de phase, $\mathrm{A}, \mathrm{B}, \mathrm{C}$ ) et en lumière réfléchie (contraste interférenciel, D, E, F); A et D, face ventrale; B et E, face latérale; C et F, face dorsale. Noter la forme régulière des polygones ventraux et la présence de ponctuations sur les bourrelets.

CHORION: Réticulations très apparentes dessinant des polygones ponctués délimités par des bourrelets épais, également ornés de ponctuations. Ces polygones présentent un fort polymorphisme selon les faces :

- polygones ventraux (fig. $2 \mathrm{~A}$ et $2 \mathrm{D}$ ) de forme régulière, pentagonale ou hexagonale, allongés parallèlement à l'axe longitudinal de l'œuf ;

- polygones latéraux (fig. $2 \mathrm{~B}$ et $2 \mathrm{E}$ ) de forme sub-losangique, à bords arrondis ou rectilignes, orientés perpendiculairement à l'axe longitudinal de l'œuf ;

- polygones dorsaux (fig. $2 C$ et $2 F$ ) plus étirés que les latéraux et orientés perpendiculairement à l'axe longitudinal de l'œuf. 
Aedes (Ochlerotatus) detritus (Haliday, 1833).

Forve: CEuf sub-cylindrique dans sa partie médiane, effilé à ses extrémités (fig. 1 C et 1 D).

TAILlE: Longueur: $0,625 \pm 0,005 \mathrm{~mm}$ (nombre de mesures 250 ; variance 0,00126). Largeur: 0,192 $\pm 0,002 \mathrm{~mm}$ (nombre de mesures 250 ; variance 0,00011).
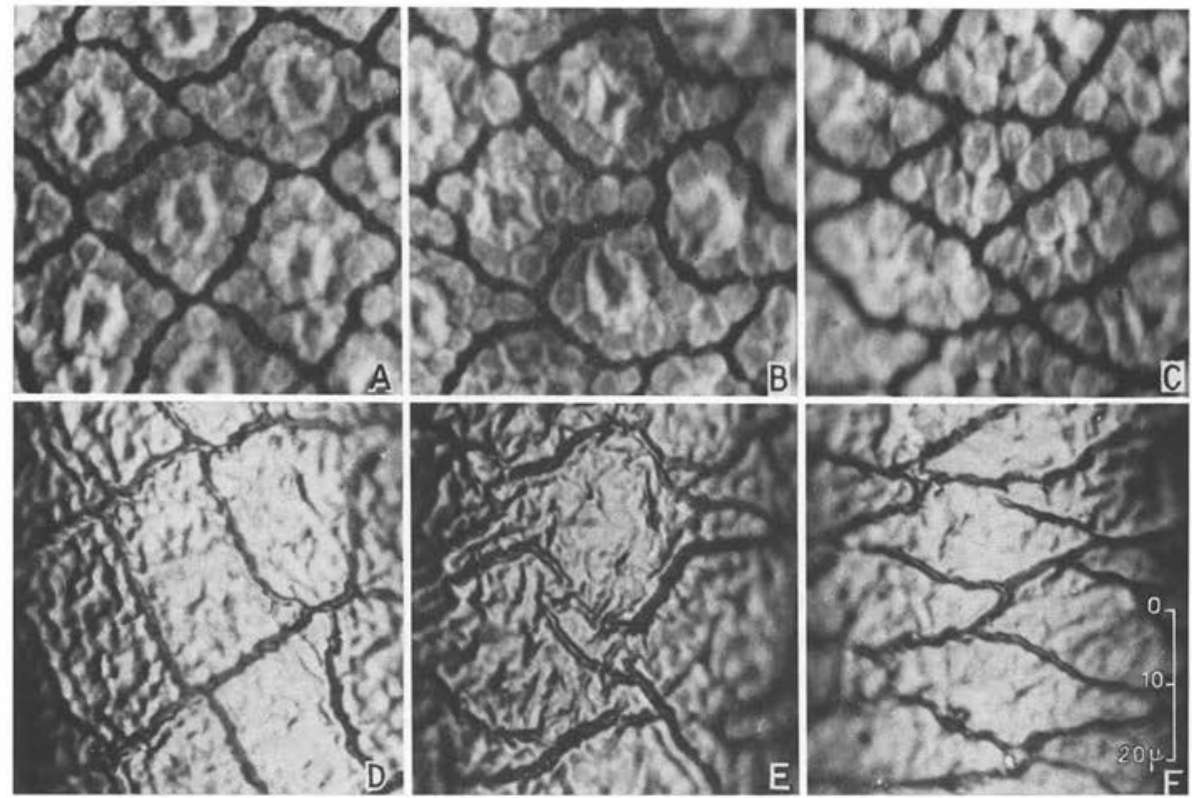

FIG. 3. - Ornementation chorionique de l'œuf chez Aedes (O.) detritus (Haliday, 1883). Vue en lumière transmise (contraste de phase, A, B, C) et en lumière réfléchie (contraste interférenciel, D, E, F); A et D, face ventrale; B et E, face latérale; C et F, face dorsale. Noter la forme «en poire» des cellules latérales et le tracé presque lisse des bourrelets.

Couleur: Noir satiné.

EXOCHORION : Mince et adhérent.

CHORION: Réticulations fines et peu apparentes dessinant des polygones finement plissés au centre et délimités par des bourrelets minces et légèrement sinueux. Comme chez Aedes caspius, la forme de ces polygones varie selon les faces:

- polygones ventraux (fig. $3 \mathrm{~A}$ et $3 \mathrm{D}$ ) grossièrement triangulaires ou quadrangulaires, quelquefois délimités par deux courbes en cloche opposées par leur base ;

- polygones latéraux (fig. $3 \mathrm{~B}$ et $3 \mathrm{E}$ ) piriformes:

- polygones dorsaux (fig. $3 C$ et $3 F$ ) sub-losangiques et étirés transversalement. 
De la courte analyse précédente, il ressort que les critères de taille et de silhouette ne présentent pas d'intérêt pour l'identification pratique des deux espèces. En revanche, la forme des polygones ornant les faces ventrales et latérales des œufs permet de les distinguer sans ambiguïté. De même la structure des bourrelets qui délimitent ces polygones est caractéristique de l'une et l'autre espèce; une telle structure est bien visible à l'objectif 28 tant en lumière transmise qu'en lumière réfléchie. En définitive, la diagnose différentielle peut être résumée comme suit:

1. - Bourrelets pourvus de ponctuations; polygones ventraux pentagonaux ou hexagonaux réguliers; polygones latéraux sub-losangiques...: Aedes (O.) caspius.

1'. - Bourrelets dépourvus de ponctuations; polygones ventraux grossièrement triangulaires ou quadrangulaires; polygones latéraux piriformes... : Aedes $(\mathrm{O}$.) detritus.

\section{Identification rapide}

En pratique courante, les œufs extraits du sol, sont alignés sur une lame porte-objet garnie de papier adhésif «double face». Ils sont ensuite identifiés en série, au microscope à contraste interférentiel (objectif 28 ou 50). Par cette technique, le tri d'une centaine d'œufs demande environ un quart d'heure si bien que le traitement complet d'un échantillon de sol est ramené, en moyenne, à une heure.

$\mathrm{Au}$ demeurant, le traitement de 500 échantillons prélevés en bordure de gîtes hébergeant les larves des deux espèces, a permis de mettre en évidence des différences appréciables dans la répartition des œufs. On note, en particulier, une ségrégation des pontes en fonction des phytocénoses (1). On peut donc espérer, pour l'avenir. une meilleure interprétation des biotopes larvaires d'Aedes caspius et d'Aedes detritus et, par le truchement d'une cartographie écologique de haute qualité, une lutte plus sélective et plus efficace.

\section{Bibliographie}

Bullock (H. R.), 1960. - Chorionic patterns of Aedes eggs by sump method. Trans. Am. Microsc. Soc., 79, 167-170.

Cousserans (J.), Gabinaud (A.), Simonneau (P.) et Sinegre (G.), 1969. - Les bases écologiques de la démoustication. Méthode de réalisation et d'utilisation de la carte phytoécologique. Vie et Milieu, 20, 1-20.

(1) L'analyse statistique et la synthèse écologique feront l'objet d'une prochaine pubíication. 
Craig (G. B.), 1955. - Preparation of the chorion of eggs of Aedine mosquitoes for microscopy. Mosquito News, 15, 228-231.

Craig (G. B.) et Horsfall (W. R.), 1956. - Taxonomic and ecological signifiance of eggs of Aedine mosquitoes. Proc. tenth int. Congr. Ent., 3, 853-857.

Craig (G. B.) et Horsfall (W. R.), 1960. - Eggs of floodwater mosquitoes. VII. Species of Aedes common in the southeastern United States (Diptera: Culicidae). Ann. Ent. Soc. Am., 53, 11-18.

FALleroni (D.), 1926. - Fauna anofelica italiana e suo « habitat ». Metodi di lotta contro la malaria. Riv. Malar., 5, 553-593.

Hinton (H. E.) et Service (M. W.), 1969. - The surface structure of Aedine eggs as seen with the scanning electron microscope. Ann. trop. Med. Parasit., 63, 409. 411.

Horsfall (W. R.), 1956. - A method for making a survey of floodwater mosquitoes. Mosquito News, 16, 66-71.

Horsfall (W. R.) et Craig (G. B.), 1956. - Eggs of floodwater mosquitoes. IV, Species of Aedes common in Illinois (Diptera: Culicidae). Ann. Ent. Soc. Am., 49, $368-$ 374.

Horsfall (W. R.), Miles (R.-C.) et SoKatch (J. T.), 1952. - Eggs of floodwater mosquitoes. I. Species of Psorophora (Diptera: Culicidae). Ann. Ent. Soc. Am., 45, 618624.

Horsfall (W.R.) et Voorhees (F. R.), 1972. - Eggs of floodwater mosquitoes. XIV. Northern Aedes (Diptera: Culicidae). Ann. Ent. Soc. Am., 65, 123-126.

Horsfall (W. R.), Voorhees (F. R.) et Cupp (E. W.), 1970. - Eggs of floodwater mosquitoes. XIII. Chorionic sculpturing. Ann. Ent. Soc. Am., 63, 1709-1716.

JAMES (S. P.), 1922. - Notes on the eggs of Culicine mosquitoes found in England. Trans. R. Soc. Trop. Med., 16, 267-269.

Kalpage (K. S.), 1968. - Mosquitoes of Manitoba. I. Description and key to Aedes eggs (Diptera: Culicidae). Can. J. Zool., 46, 699-718.

MYERS (C. M.), 1967. - Identification and description of Aedes eggs from California and Nevada (Diptera: Culicidae). Can. Ent., 99, 795-807.

Rıoux (J. A.), 1958. - Les Culicides du « midi » méditerranéen. Le Chevalier, édit., (Paris), 303 p. 
Rioux (J.-A.), Croset (H.), Corre (J.-J.), Simonneau (P.) et Gras (G.), 1967, - Les bases phytoécologiques de la lutte anticulicidienne. Cartographie des biotopes larvaires. Ses applications opérationnelles dans le «midi» méditerranéen. Ann. Parasit. hum. comp., 42, 665-680.

Rioux (J.-A.), Croset (H.), Corre (J.-J.), Simonneau (P.) et Gras (G.), 1968, - Phytoecological basis of mosquito control : cartography of the larval biotopes. Mosquito News, 28, 572-582. 